\title{
Membrane Metalloendopeptidase (MME) Suppresses Metastasis of Esophageal Squamous Cell Carcinoma (ESCC) by Inhibiting FAK-RhoA Signaling Axis
}

Mengqing Li, ${ }^{*}$ Ling Wang, ${ }^{*}$ Yuting Zhan, ${ }^{*}$ Tingting Zeng, ${ }^{*}$ Xu Zhang, ${ }^{* \dagger}$ Xin-Yuan Guan, ${ }^{*}$ and Yan $\mathrm{Li}^{*}$

From the State Key Laboratory of Oncology in South China, * Collaborative Innovation Center for Cancer Medicine, Sun Yat-sen University Cancer Center, Guangzhou; the Department of Surgery, ${ }^{\dagger}$ Sun Yat-sen University Cancer Center, Guangzhou; and the Department of Clinical Oncology, ${ }^{\ddagger}$ The University of Hong Kong, Hong Kong, China

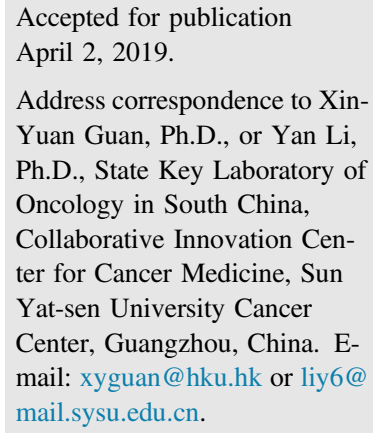

\begin{abstract}
Esophageal squamous cell carcinoma (ESCC) is a typical neoplastic disease and a frequent cause of death in China. Although great achievements have been made in diagnostic strategies and combination therapies in recent years, the prognosis of ESCC is still poor. Metastasis/recurrence has been the major factor responsible for poor prognosis. However, the underlying mechanism of ESCC dissemination remains elusive. Membrane metalloendopeptidase (MME) is a transmembrane glycoprotein that degrades a number of substrates. This study's results indicated that the down-regulation of MME is significantly associated with advanced clinical stage $(P<0.05)$ and lymph node metastasis $(P<0.05)$. The down-regulation of MME in ESCC tumor tissues is correlated to poorer prognosis of the patients. Functional studies demonstrated that MME could significantly inhibit ESCC tumor cell metastasis in vitro and in vivo. MME overexpression could also interrupt ESCC tumor cell adhesion. Mechanistically, MME inhibits the phosphorylation of FAK thus interrupting the FAK-RhoA axis, which is important in cell movement. Taken together, these data show that MME regulates ESCC via FAK-RhoA axis. High expression of MME may indicate a beneficial outcome for patients. (Am J Pathol 2019, 189: 1462-1472; https://doi.org/10.1016/j.ajpath.2019.04.007)
\end{abstract}

Esophageal squamous cell carcinoma (ESCC), along with esophageal adenocarcinoma, is the major subtype of esophageal cancer recognized, according to microanatomic categorization. In Asia, ESCC is the predominant form, which accounts for $57 \%$ of diagnoses compared with $18 \%$ in the United States. ${ }^{1}$ It is also the fourth leading cause of cancer-related deaths in China. ${ }^{2}$ Eastern Asia has the highest incidence of esophageal cancer, whereas it is lowest in Western Africa. ${ }^{3}$ Although the incidence rates from 2000 to 2011 decreased for ESCC, and scientists have performed numerous studies to identify prognostic markers, the outcome for ESCC patients remains grim. Thus, understanding the detailed molecular mechanisms in ESCC progression and developing novel strategies for treatment are urgently needed to improve the survival rates for these sufferers.
The membrane metalloendopeptidase $(M M E)$ gene is located at human chromosome 3q21-27. It encodes a 100$\mathrm{kD}$ type II transmembrane glycoprotein, ${ }^{4}$ a widely expressed membrane metalloendopeptidase that degrades a number of substrates. The active site of the enzyme faces the extracellular space. ${ }^{5} \mathrm{MME}$ is widely expressed in a broad range of tissues, and in kidney and lung tissues, it has particularly abundant expression. ${ }^{6}$

The molecule was first identified as a tumor-specific antigen (common acute lymphoblastic leukemia antigen) in leukemia and has been used for diagnosing B-lineage

Supported by NSFC grants 81672357,81772554 , and 81871903 , National Key R\&D Program of China grant 2017YFC1309000, GDSTP grant 2017A020215179, and SUMSIP grant 16ykjc34.

Disclosures: None declared. 
acute lymphoblastic leukemia in combination with other B-lineage markers. ${ }^{7}$ Its expression can indicate prognosis in acute lymphoblastic leukemia. ${ }^{8}$ Although researchers have observed associations between MME expression and a few types of cancer, the specific association remains obscure. Interestingly, in some cancers, such as colorectal carcinoma, pancreatic endocrine tumor, and advanced melanomas, MME is overexpressed. ${ }^{9}$ In some types of tumors, most notably lung cancers and ovarian cancer, MME is down-regulated. In prostatic carcinoma, MME is expressed in androgen-sensitive LNCaP cells and possesses tumor suppressive ability. ${ }^{10}$

MME not only is a cell surface marker, but also has a critical role in regulating many biological processes. ${ }^{11}$ However, the role of MME has not been investigated in the ESCC oncogenesis and progression. In this study, the clinical relevance of MME expression was investigated in human ESCC samples. MME down-regulation was correlated with poorer prognosis of ESCC patients. Also, MME introduction inhibited tumor cell metastasis, while not affecting tumor cell proliferation. The underlying mechanism of how MME inhibits ESCC tumor cell was also explored.

\section{Materials and Methods}

\section{Cell Lines and Cell Culture}

The HKESC1, EC18, and EC109 cell lines were kindly provided by Prof. G. Srivastava (Department of Pathology, The University of Hong Kong, Hong Kong, China). An immortalized nonmalignant esophageal cell line was provided by Prof. G.S. Tsao (Department of Anatomy, The University of Hong Kong). ESCC cell lines (KYSE520, KYSE510, KYSE410, KYSE180, KYSE140, and KYSE30) were acquired from the German Biological Resource Center (DSMZ, Braunschweig, Germany). 293FT cell were obtained from Invitrogen (Thermo Fisher Scientific, Waltham, MA). ESCC cell lines were cultured in Dulbecco's modified Eagle's medium with $10 \%$ supplements of fetal bovine serum Gibco, Thermo Fisher Scientific. The cells were authenticated by short tandem repeat profiling.

\section{Clinical Specimens, ESCC Tissue Microarray, and Immunohistochemical Analysis}

The study was performed under the review of the Committees for Ethical Review of Research Involving Human Subjects, both in the Sun Yat-sen University Cancer Center and Zhengzhou University.

The ESCC tumor tissues along with the adjacent nontumor parts (at least $5 \mathrm{~cm}$ away from the tumor site) were obtained from Linzhou Cancer Hospital, and the tissue microarray containing 300 pairs of tumor specimens and the corresponding non-tumor tissues were constructed. $^{12}$

The paraffin wax of the specimens was removed by xylene, then the slides were washed in a series of graded alcohol: $100 \%, 95 \%, 75 \%$, and $50 \%$ for rehydration and then incubated with $3 \%$ hydrogen peroxide. For antigen retrieval, the slides were boiled in EDTA buffer for 30 minutes. Five percent bovine serum albumin in phosphatebuffered saline (PBS) was used to block the nonspecific binding. After incubation with primary antibody against MME (PB0026, Boster Biological Technology, Wuhan, China) at $4^{\circ} \mathrm{C}$ overnight, the slides were washed and incubated with horseradish peroxidase-conjugated secondary antibody before stained with the DAB substrate (Dako, Glostrup, Denmark). An immunoreactivity pathological score system which is widely used was applied as previously described. ${ }^{12}$ In brief, the proportion of MME stained cells from $0 \%$ to $100 \%$ ( $<5 \%, \geq 5 \%$ to $<25 \%$, $\geq 25 \%$ to $<50 \%, \geq 50 \%$ to $<75 \%, \geq 75 \%$ to $100 \%$ ) got a proportion score (PS) of 0 to 4 . The intensity of MME staining was also scored as 0 to 4 for different degrees of staining, from negative to strong. The staining index (SI) was calculated using a product formula: positive percentage $\times$ intensity to evaluate the score. Because the average SI of nontumor tissues $\left(\mathrm{N}_{\mathrm{SI}}\right)$ was equal to 5.2 , the down-regulation of MME was determined as $\mathrm{T}_{\mathrm{SI}}<5$.

\section{Plasmids, shRNAs, and Primers}

The recombinant construct pLV105-MME and vector control were bought from GeneCopia (Rockville, MD). Lentivirus was obtained by the cotransfection of recombinant constructs with three lentivirus packaging mix (pLp1, pLp2, and pLp-VSVG) (Invitrogen) into 293FT (Invitrogen). ESCC cells were infected with lentivirus and stably overexpressed cells were established by selecting with $5 \mu \mathrm{g} / \mathrm{mL}$ of puromycin for about 14 days.

The shRNA products were purchased from Genechem (Shanghai, China). The knockdown efficiency was tested by quantitative RT-PCR and Western blot. The primers and shRNAs are listed in Table 1.

\section{Table 1 Primers and shRNAs}

\begin{tabular}{|c|c|}
\hline Primer name & Sequence \\
\hline MME & F: 5'-AGTCGGAAACTGGCAGATAGC-3' \\
\hline MME & R: 5'-GGTAGTGTTGTACTGGGCCAAT-3' \\
\hline$\beta$-Actin & F: 5'-CTCCATCCTGGCCTCGCTGT-3' \\
\hline$\beta$-Actin & R: 5'-GCTGTCACCTTCACCGTTCC-3' \\
\hline$\beta$-Tubulin & F: 5'-GAGCTGTTCAAGCGCATCTC-3' \\
\hline$\beta$-Tubulin & R: 5'-TCCTCCTCGTCGTCTTCGTA-3' \\
\hline \multicolumn{2}{|c|}{ shRNAs targeting MME } \\
\hline Sh1 & 5'-TCCAGGAGTATGTCACCTT-3' \\
\hline Sh2 & 5'-CAAGGCTGATGGAAGACTT-3' \\
\hline Sh3 & $5^{\prime}$-GGTGAACTTCCAGGAGTAT-3' \\
\hline
\end{tabular}

F, forward; $R$, reverse. 


\section{Immunofluorescence (IF) Assay}

Cell fixation with $4 \%$ paraformaldehyde was followed by permeabilization with $0.1 \%$ Triton $\mathrm{X}-100$. After incubating with primary antibodies (rabbit anti-FAK, Cell Signaling Technology, Danvers, MA) at $4^{\circ} \mathrm{C}$ overnight, cells were washed and incubated with fluorescent secondary antibody for 1 hour. The cells were counterstained with DAPI (Thermo Fisher Scientific) for 3 minutes at room temperature.

For F-actin staining, direct immunofluorescence of fluorescein isothiocyanate-conjugated phalloidin (SigmaAldrich, St. Louis, MO) was applied at $1 \%$ in PBS to stain the fixed cells for 30 minutes. The slides were then mounted in VECTASHIELD with DAPI (Vector Laboratories, Burlingame, CA). ${ }^{13}$ All images were visualized under Olympus FLUOVIEW FV1000 confocal laser scanning microscope or OLYMPUS BX53 fluorescent microscope (Olympus Corporation, Tokyo, Japan).

\section{Wound Healing}

A $10-\mu \mathrm{L}$ pipette tip was used to scratch a straight wound in the experimental group cells or the control cells in $35-\mathrm{mm}$ dishes. The migration photos were captured at 0,24 , or 48 hours after scratching to compare the gap closure speed.

\section{Cell Invasion and Migration Assays}

Two kinds of 24-well chambers were used in the assays (BD Biosciences, Franklin, NJ), both of them have $8-\mu \mathrm{m}$ microporous membranes to separate the chambers. In the invasion assay, the membranes were coated with matrix gel.

Tumor cells were seeded in the top compartment with serum-free medium. The bottom compartments were filled with Dulbecco's modified Eagle's medium with $10 \%$ fetal bovine serum as the chemoattractant. After 18 or 28 hours, the cells that migrated through the pores were fixed. The cells were stained with crystal violet, observed, and then quantified in over 10 fields under the microscope.

\section{Foci Formation Assay}

Cells, $1.0 \times 10^{3}$ per well, were seeded in 6-well plates. After 10-day culture, colonies that formed were stained using crystal violet (Sigma-Aldrich) and counted. Three independent experiments were repeated.

\section{Animal Experiments}

The animal experiments were approved by the Institutional Animal care and Use Committee of Sun Yat-sen University Cancer Center. Cell proliferation in vivo was evaluated by the subcutaneous tumor models. Briefly, MME overexpressed cells and vector control cells were suspended in PBS, then subcutaneously implanted in the lateral flank of 4-week-old BALB/c nude mice $(n=5)$. Tumor size of the mice was measured every 7 days. Tumor volume was calculated as: $\mathrm{V}=\mathrm{L} \times \mathrm{W}^{2} \times 0.5\left(\mathrm{~mm}^{3}\right)$.

At the end of the experiment, the animals were sacrificed, and tumors were weighed.

To establish metastasis models, a total of $5 \times 10^{5}$ EC109 or K510 derivative cells were injected via the tail vein of $\mathrm{Nu} / \mathrm{Nu} \mathrm{BALB} / \mathrm{c}$ mice (4-week-old, female, $n=5$ for each group). After 2 months, the animals were sacrificed. The animals' lungs were isolated and fixed and the number of nodules was counted. The lung sections were examined by hematoxylin and eosin stain.

For the lymph node metastasis model, cells in PBS solution $\left(1.5 \times 10^{5} / 20 \mu \mathrm{L}\right)$ were injected into one hind foot per animal. The mice were euthanized 2 months later. The popliteal lymph nodes were excised and fixed. Each experimental group consisted of five $\mathrm{Nu} / \mathrm{Nu} \mathrm{BALB} / \mathrm{c}$ mice.

\section{Western Blot and Antibodies}

Cells were lyzed with radioimmunoprecipitation assay buffer (Cell Signaling Technology) containing protease and phosphatase inhibitor (Roche, Indianapolis, IN). The antibodies were: $\beta$-tubulin, $\beta$-actin, FAK, P-FAK (Y397), ERK, P-ERK (Thr202/204), MEK, and P-MEK (Ser217/221) (Cell Signaling Technology), and Pan-CK (Zhongshan Golden Bridge Bio-technology, Beijing, China).

\section{Adhesion Assay}

The 24-well plate was first coated with fibronectin and washed twice with PBS before plating cells. MME- or vector-transfected cells were digested to prepare suspensions containing $2 \times 10^{5}$ cells $/ \mathrm{mL}$ in Dulbecco's modified Eagle's medium $+3 \%$ fetal bovine serum. The cell suspension $(500 \mu \mathrm{L})$ was added and allowed to attach for 2, 4, or 8 hours at $37^{\circ} \mathrm{C}$. Unattached cells were then gently removed. Ultimately, the cells were stained by crystal violet and observed under the microscope. All of the experiments were repeated three times in duplicate wells. ${ }^{14}$

\section{RhoA Activation Assay}

To assess the potential contribution of GTPases to MMEinduced inhibition of ESCC cell motility, the levels of active RhoA in cells were measured by GST-rhotekin pulldown assays according to the manufacturer's directions (Millipore, Denver, CO). ${ }^{15}$ In brief, MME- or vector-transfected cells were selected by puromycin and then starved in medium without fetal bovine serum overnight before treatment. Cells were harvested in $\mathrm{Mg}^{2+}$ lysis buffer, clarified by centrifugation. Fifteen to $23 \mu \mathrm{L}$ (20 to $30 \mu \mathrm{g}$ ) of the Rho Assay Reagent slurry were added to the cell extract. After washing 3 times, the agarose beads were resuspended in $25 \mu \mathrm{L}$ of $2 \times$ Laemmli reducing sample buffer, then boiled for 5 minutes. 


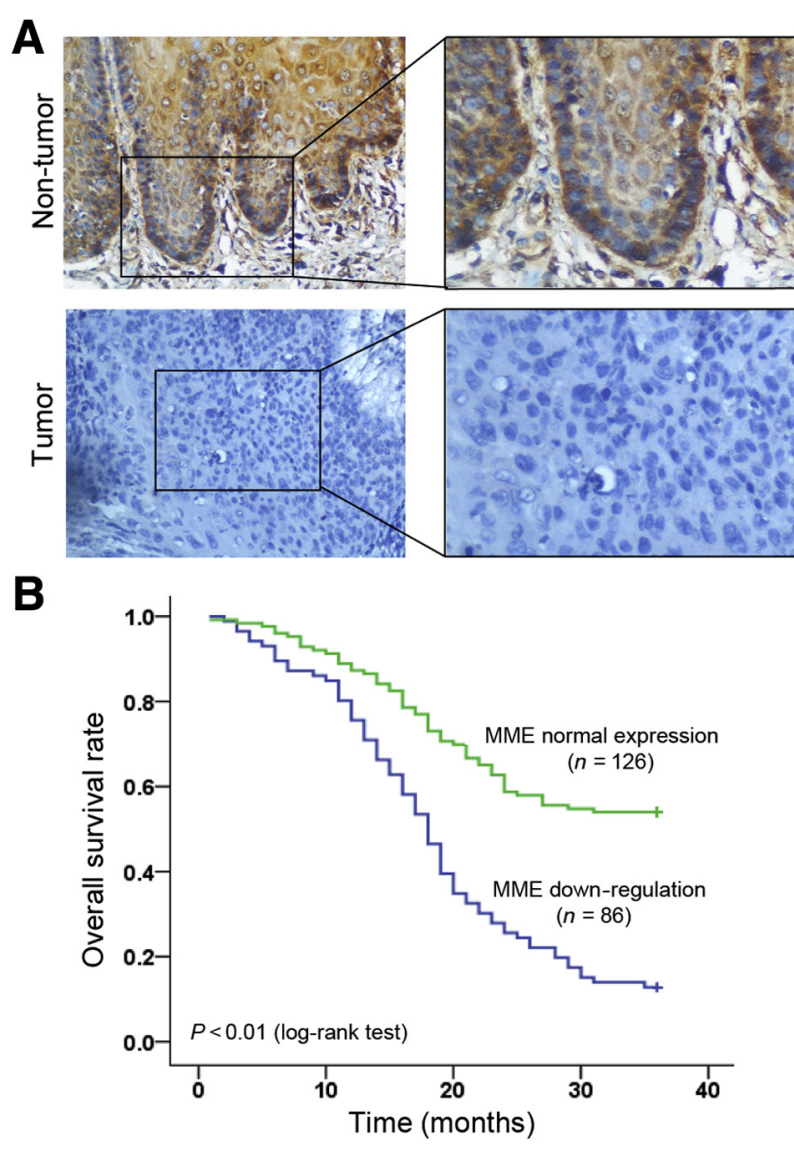

Figure 1 Down-regulation of membrane metalloendopeptidase (MME) in esophageal squamous cell carcinoma (ESCC) is associated with poorer outcomes. A: Representative immunohistochemical staining of MME expression in matched primary ESCC sample and its corresponding nontumorous tissue. Boxed areas are shown at higher magnification in the images on the right. B: Kaplan-Meier curves for overall survival rate of patients with ESCC according to the expression status of MME. Original magnification: $\times 20$ (A, left column); $\times 40$ (A, right column). $P<0.05$.

Activated RhoA of equal amounts of total protein was detected by Western blotting in each sample. All steps were performed at $4^{\circ} \mathrm{C}$ or on ice to reduce hydrolysis.

Samples were also analyzed to verify that MME knockdown did not have secondary effects on RhoA expression. After normalization of the results to the control samples, the results of three independent experiments were combined and analyzed.

\section{Statistical Analysis}

All results were presented in forms of means \pm SD. Oneway analysis of variance tests were applied for comparisons of the statistical differences among more than two groups. Other statistical differences were evaluated via the unpaired $t$-tests. The $\chi^{2}$ test was performed to evaluate the association between the clinicopathological parameters and MME levels. Survival curves were plotted using Kaplan-Meier analysis and log rank test was adopted.
Table 2 Correlation of MME Expression with Clinicopathological Features in ESCC Patients

\begin{tabular}{|c|c|c|c|}
\hline Features & Total & $\begin{array}{l}\text { MME } \\
\text { down-regulation* }\end{array}$ & $\begin{array}{l}P \\
\text { value }\end{array}$ \\
\hline \multicolumn{4}{|l|}{ Sex, $n(\%)$} \\
\hline Male & 113 & $50(44.2 \%)$ & \multirow[t]{2}{*}{0.152} \\
\hline Female & 99 & $36(36.4 \%)$ & \\
\hline \multicolumn{4}{|c|}{ Age in years, $\mathrm{n}(\%)$} \\
\hline$\leq 60$ & 95 & $36(37.9 \%)$ & \multirow[t]{2}{*}{0.284} \\
\hline$>60$ & 117 & $50(42.7 \%)$ & \\
\hline \multicolumn{4}{|c|}{ Differentiation, $n(\%)$} \\
\hline Well/moderate & 189 & $71(40.7 \%)$ & \multirow[t]{2}{*}{0.535} \\
\hline Poor & 23 & $15(39.1 \%)$ & \\
\hline \multicolumn{4}{|l|}{ Tumor stage, $\mathrm{n}(\%)$} \\
\hline I-II & 119 & $41(34.4 \%)$ & \multirow[t]{2}{*}{0.040} \\
\hline III-IV & 93 & $45(48.4 \%)$ & \\
\hline \multicolumn{4}{|c|}{ Lymph node metastasis, $n(\%)$} \\
\hline NO & 116 & $39(33.6 \%)$ & \multirow[t]{2}{*}{0.024} \\
\hline N1 & 96 & $47(49.0 \%)$ & \\
\hline
\end{tabular}

${ }^{*}$ MME down-regulation was defined as $\mathrm{T}_{\mathrm{SI}}<5$.

Using the Cox proportional hazards model, the significance of various clinicopathological variables for survival was analyzed. $P$-value of less than 0.05 was considered significant.

\section{Results}

\section{Down-Regulation of MME in ESCC Specimens Is Correlated to Poorer Prognosis of the Patients}

The TMA was stained with MME and the correlation analysis of MME expression with clinicopathological features was performed with 212 informative ESCC tumor cases. Noninformative samples were not taken into account: few tumor cells in the sample, unrepresentative samples or lost samples. The average staining index of MME in nontumor tissues was 5.2 and the down-regulation of MME was determined as SI $<5$. In 212 valid ESCC tumor tissues, as the results showed, 86 cases (40.5\%) showed down-regulation of MME (Figure 1A). The correlation between MME level and clinicopathological features of patients with ESCC was studied next. MME down-regulation was significantly associated with lymph node metastasis (Pearson $\chi^{2}$ test, $P<0.05$ ), advanced clinical stage (Pearson $\chi^{2}$ test, $P<0.05$ ) (Table 2). Kaplan-Meier survival analysis revealed that patients with MME down-expression underwent poorer overall survival $(P<0.01)$ (Figure 1B). Univariate and multivariate survival analyses were also achieved using the Cox regression model. Univariate analysis demonstrated that down-regulation of $\operatorname{MME}(P<0.01)$, along with poor differentiation $(P=0.013)$ and lymph node metastasis, was considered a significant prognostic factor for overall survival (Table 3). Multivariate analysis taking into account the effects of all variables possible also proved that for ESCC patient overall survival, MME down-regulation was an independent prognostic factor. 
Table 3 Univariate and Multivariate Analysis of Different Prognostic Variables in Patients with ESCC

\begin{tabular}{|c|c|c|c|c|c|c|}
\hline \multirow[b]{2}{*}{ Features } & \multicolumn{3}{|c|}{$\underline{U n i v a r i a t e}$ analysis* } & \multicolumn{3}{|c|}{ Multivariate analysis* } \\
\hline & $\mathrm{HR}$ & $95 \% \mathrm{CI}$ & $P$ value & $\mathrm{HR}$ & $95 \% \mathrm{CI}$ & $P$ value \\
\hline Sex & 1.140 & $0.809-1.606$ & 0.454 & NA & NA & NA \\
\hline Differentiation & 0.403 & $0.197-0.824$ & 0.013 & 0.365 & $0.178-0.750$ & 0.006 \\
\hline LN metastasis & 2.189 & $1.547-3.097$ & $<0.001$ & 2.065 & $1.456-2.927$ & $<0.001$ \\
\hline MME down-regulation & 0.348 & $0.246-0.493$ & $<0.001$ & 0.365 & $0.256-0.518$ & $<0.001$ \\
\hline
\end{tabular}

${ }^{*}$ Cox regression model.

HR, hazard ratio; LN, lymph node; NA, not applicable.

\section{Overexpression of MME Suppresses Tumor Cell Mobility and Metastasis}

MME protein was detected in nine ESCC cell lines and NE1 (an immortalized normal esophageal epithelial cell line) using Western blotting. MME showed frequent down-regulation in the majority of ESCC cell lines in comparison with NE1 (Figure 2A).

EC109 and KYSE510 cells with MME stable expression were established by lentiviral transduction (Figure 2B). Foci formation results showed that $\mathrm{MME}$ overexpression in EC109 could mildly decrease the tumorgenicity, whereas in KYSE510, it has no significant influence on cell growth in vitro and in vivo (Supplemental Figure S1). Woundhealing assay showed that MME-transfected cells obtained slower migration rate compared with the control cells (Figure 2C). Cells' ability to migrate and invade significantly decreased with MME overexpression $(P<0.01)$ (Figure 2D). MME was also stably knocked down in KYSE180 and KYSE410 using shRNAs. Gene silencing
A

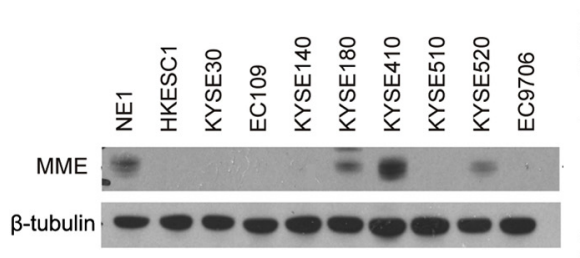

C
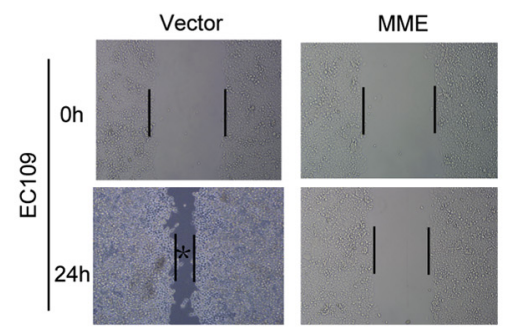

D

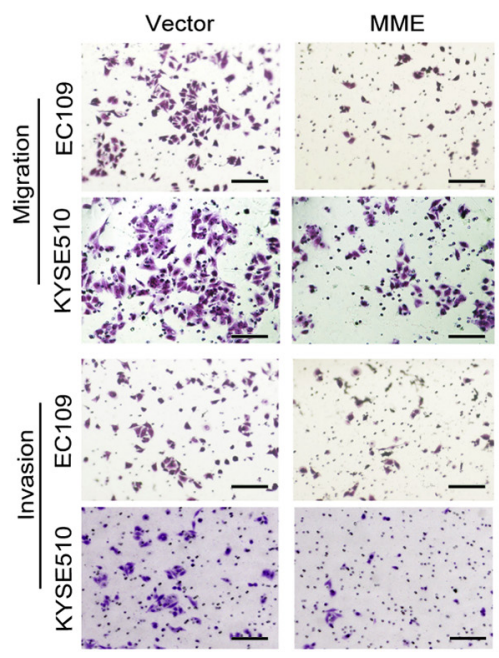

B
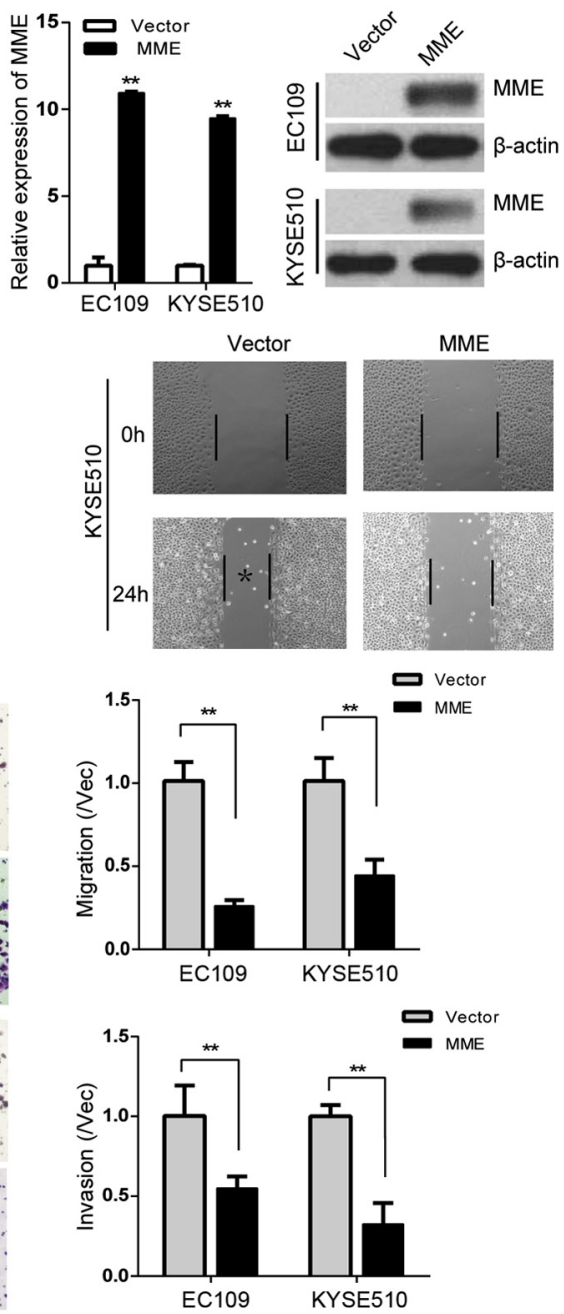

Figure 2 Overexpression of membrane metalloendopeptidase (MME) suppresses esophageal squamous cell carcinoma (ESCC) cell motility in vitro. A: Western blot analysis of MME expression in an immortalized esophageal epithelial cell line (NE1) and ESCC cell lines. $\beta$-Tubulin was used as a loading control. B: MME expression was evaluated by real-time quantitative PCR and Western blotting in MME- and vector-transfected cells. $\beta$-Actin was used as a loading control. C: Wound-healing assay shows that introduction of MME inhibits tumor cell migration. Representative images were taken at 0 or 24 hours after scratching. Black lines indicate the front of cell migration. D: Representative pictures and summary of migrated and invaded EC109 and KYSE510 derivative cells (MME and vector control). Data are expressed as means \pm SD. $n=3$ independent experiments. ${ }^{*} P<0.05,{ }^{*} P<0.01$ versus vector (independent $t$-test). Scale bars $=$ $100 \mu \mathrm{m}$. Original magnification, $\times 100$ (C). 
was confirmed at both the RNA and protein level (Figure 3, $\mathrm{A}$ and $\mathrm{B})$. In vitro functional assays also found silencing MME could enhance wound healing closure (Figure 3C), cell migration, and invasion ability (Figure 3D).

\section{Overexpression of MME Interrupts Cell Adhesion and FAK Activation}

It was next investigated whether MME had an effect on cell adhesion to the matrix as it is an important stage for cancer cell metastasis. Cell-cell adhesiveness is generally reduced in human cancers, but cell-matrix junctions that form firm adhesions increased. ${ }^{16,17}$ The results demonstrated that MME overexpression significantly decreased cell adhesion to the plate (Figure 4, A and B), which is not good for tumor cell metastasis.

Because previous studies reported that MME could inhibit FAK phosphorylation, and FAK happens to be an essential nonreceptor tyrosine kinase regulating cell adhesive signaling and migrating, ${ }^{18,19}$ phosphorylated FAK protein level decreased in the MME overexpressing cells (Figure 4C). The results were further confirmed using immunofluorescence, which showed that the immunoreactivity against phosphorylated FAK was markedly weakened compared with the vector control, both in EC109 and KYSE510 (Figure 4D). The protein levels of MME and phosphorylated FAK were also detected in ESCC tumor tissues and paired nontumor tissues. Reciprocal correlation between MME and phosphorylated FAK was observed in 6 of $10 \mathrm{ESCC}$ cases (Figure 4E).

\section{MME Suppresses FAK-RhoA Axis}

Recent studies proved that FAK is responsible for RhoA activity. ${ }^{20}$ It is indicated that RhoA activity has close associations with the actin reorganization, namely stress fiber formation, in epithelial cells, which also plays a vital part in cell metastasis. The RhoA activity assay indicated that the
A

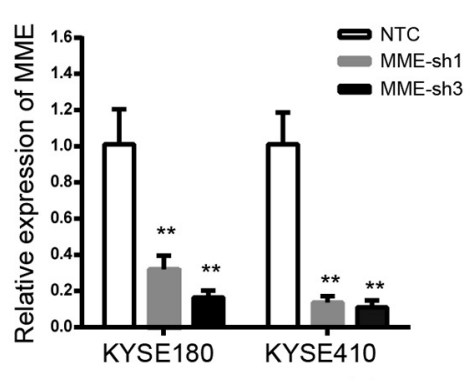

C

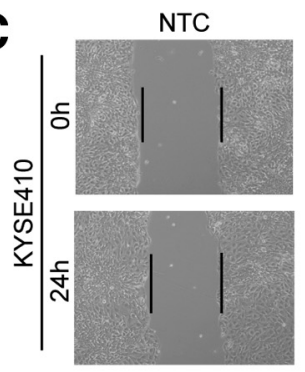

D
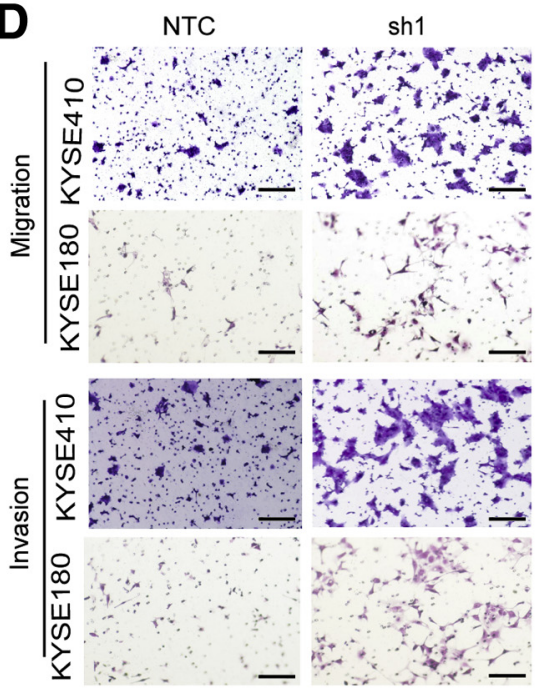

B
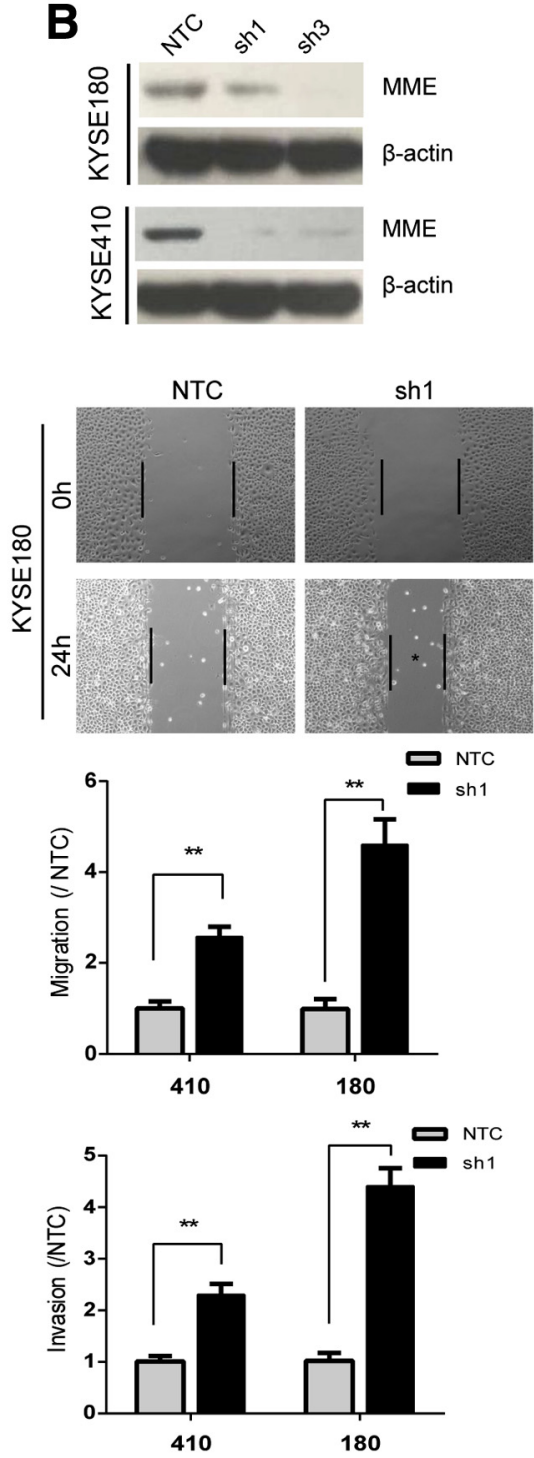

Figure 3 Knocking down membrane metalloendopeptidase (MME) promotes cell motility. A and B: Down-regulation of MME is detected by real-time quantitative PCR (A) and Western blotting (B) in KYSE180 and KYSE410 derivative cells. $\beta$-Actin was used as a loading control. C: Woundhealing assay shows that silencing MME enhances tumor cell migration. Representative images were taken at 0 or 24 hours after scratching. Black lines indicate the front of cell migration. D: Representative pictures and summary of migrated and invaded KYSE140 and KYSE180 derivative cells (sh1 and nontarget control). Data are expressed as means \pm SD. $n=3$ independent experiments. ${ }^{*} P<0.05,{ }^{*} P P<0.01$ versus NTC (independent $t$-test). Scale bars $=100 \mu \mathrm{m}$. Original magnification, $\times 100$ (C). NTC, nontarget control. 

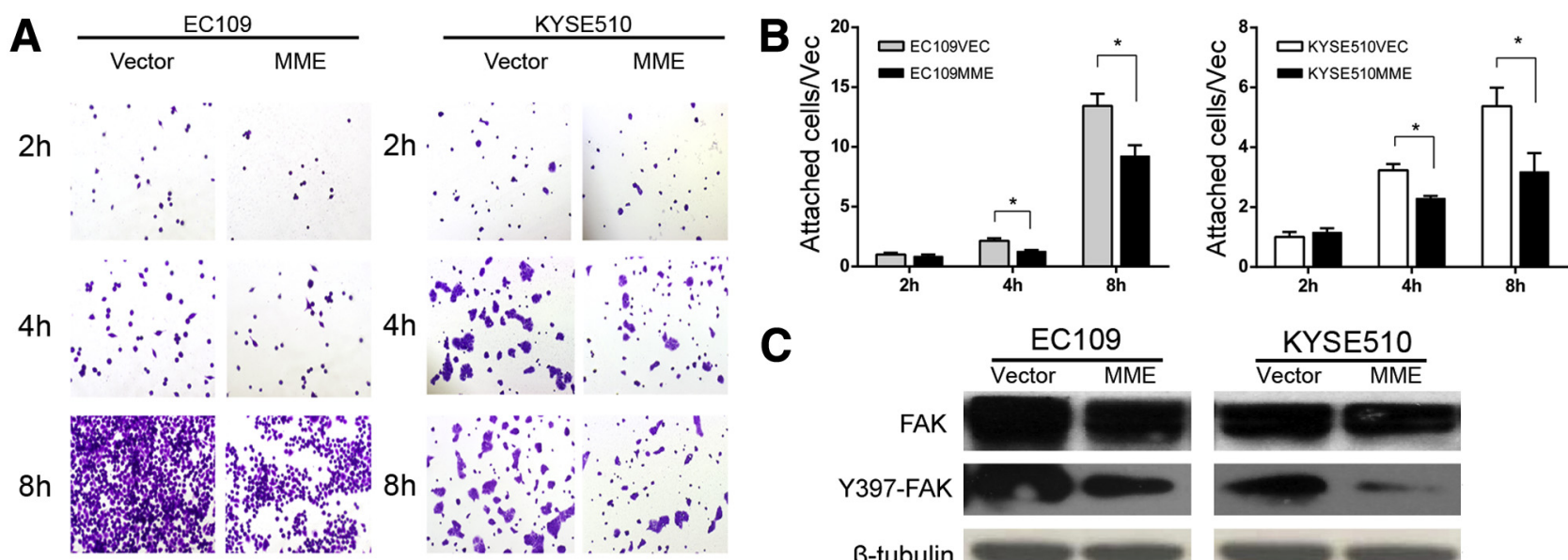

C
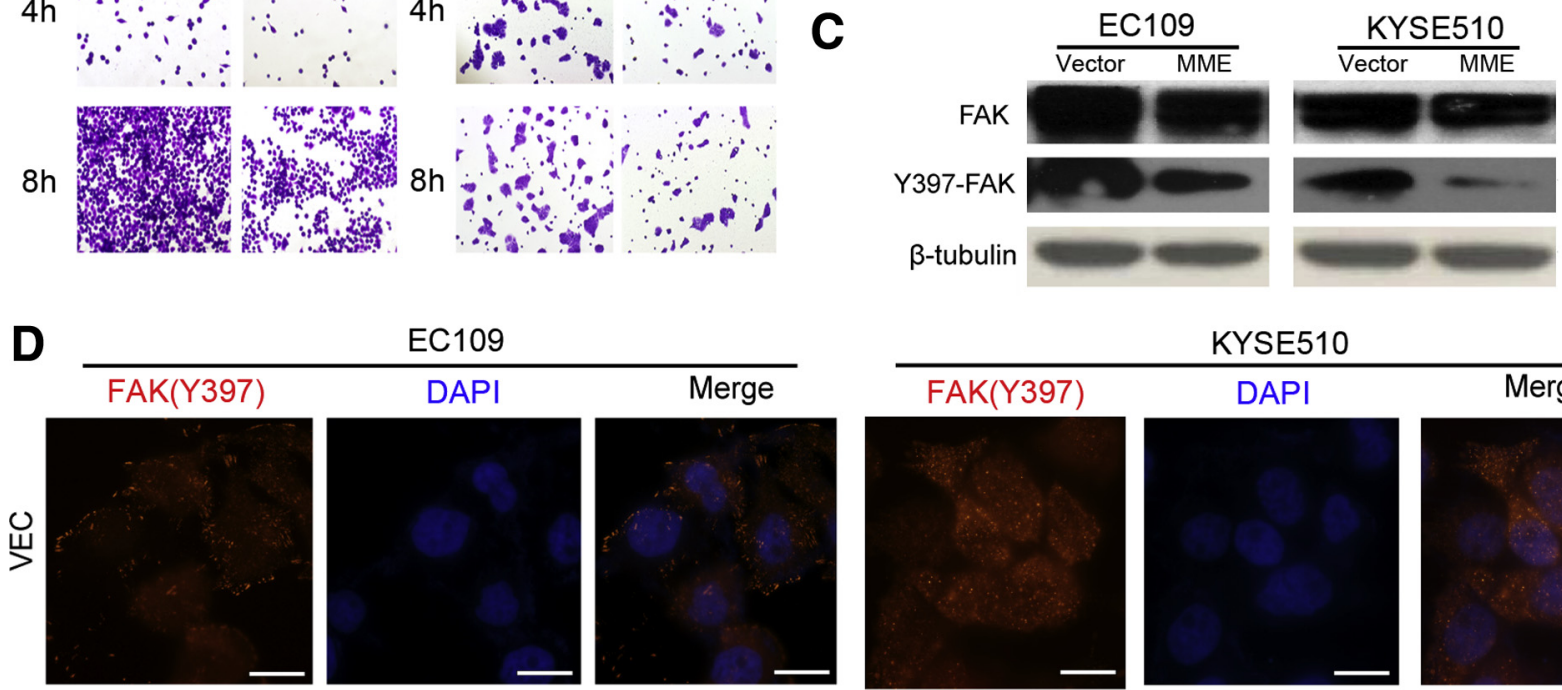

EC109
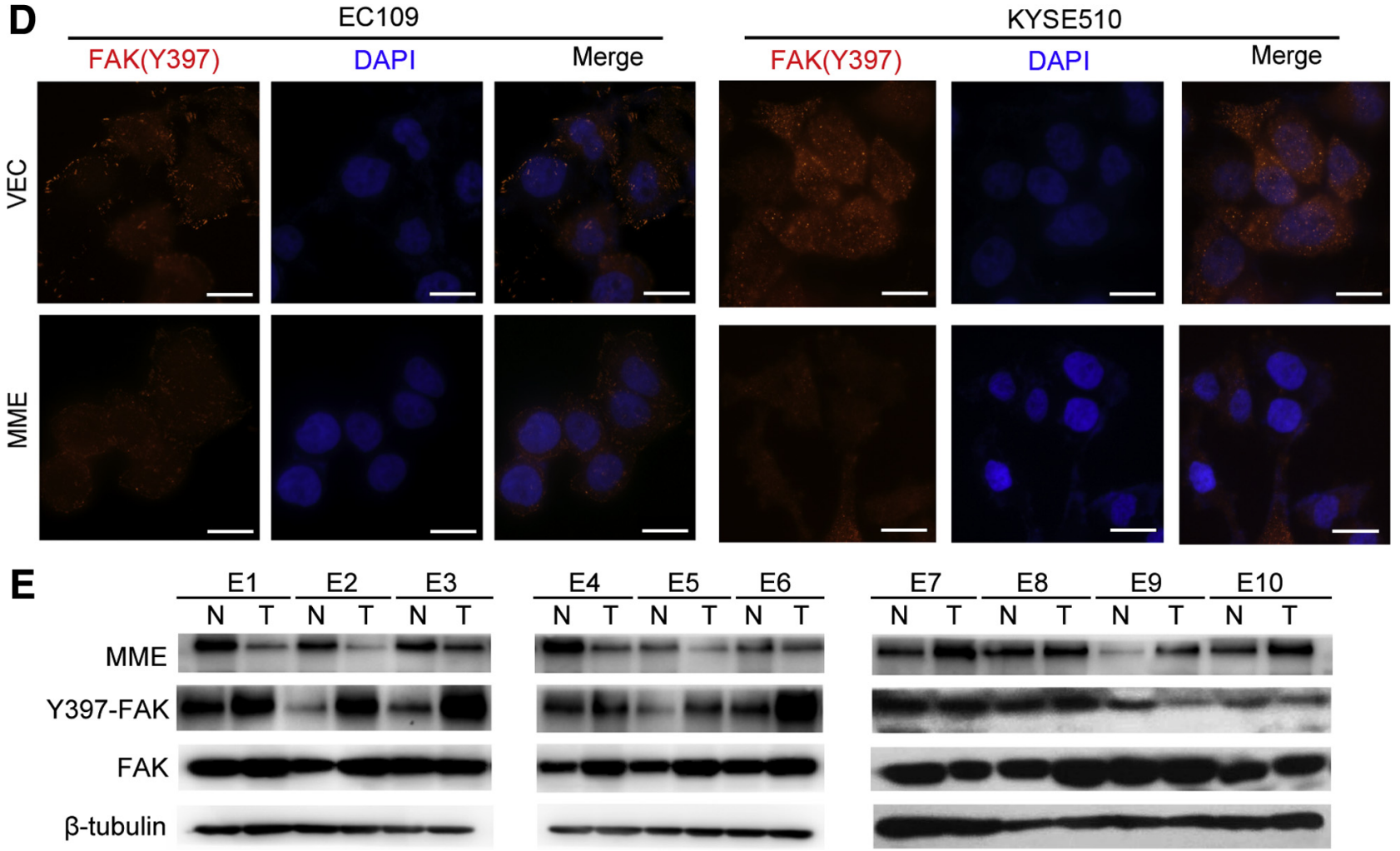

Figure 4 Membrane metalloendopeptidase (MME) suppresses cell adhesion and FAK-RhoA axis. A and B: Representative pictures (A) and summary (B) show different adhesive properties of MME and VEC cells after cells were starved. The cells were allowed to attach to fibronectin-coated plates for 2, 4, or 8 hours at $37^{\circ} \mathrm{C}$. Attached cells were fixed, stained, and then counted under the microscope. C: Western blot analysis of p-FAK and FAK expression in MME overexpressed esophageal squamous cell carcinoma (ESCC) cells and vector control cells. $\beta$-Tubulin was used as a loading control. D: Immunofluorescence staining for p-FAK (red) in MME- or vector-transfected cells. Nuclei were counterstained with DAPI (blue). E: The protein levels of MME, p-FAK, and FAK were determined in ESCC clinical samples. $\beta$-Tubulin was set as a loading control. Reciprocal correlation between MME and p-FAK is observed in six ESCC cases: E1, E2, E3, E5, E9, and E10. ${ }^{*} P<0.05$. Scale bars $=20 \mu \mathrm{m}$. Original magnification, $\times 100(\mathbf{A}) . \mathrm{N}$, precancerous lesions; $\mathrm{T}$, cancerous tissue.

MME overexpression dramatically suppressed the activity of RhoA in EC109 and KYSE510 cells, whereas silencing MME increased GTP-RhoA in KYSE410 and KYSE180 cells (Figure 5A).

As is well known, the arrangement of the actin cytoskeleton can significantly influence cell morphology. This characteristic offers us insight into further discovering the signaling pathway of migrating or attached cells. With fluorescein isothiocyanate-phalloidin staining, the stress fiber formation was found to be decreased in MME overexpressed cells compared with the control cells (Figure 5B).

The downstream pathway signaling of FAK-RhoA was next studied, and it was found that phosphorylated Erk1/2 (Thr202/204) and MEK1/2 (Ser217/221) decreased in MME overexpressed EC109 and KYSE510 cells (Figure 5C). The 


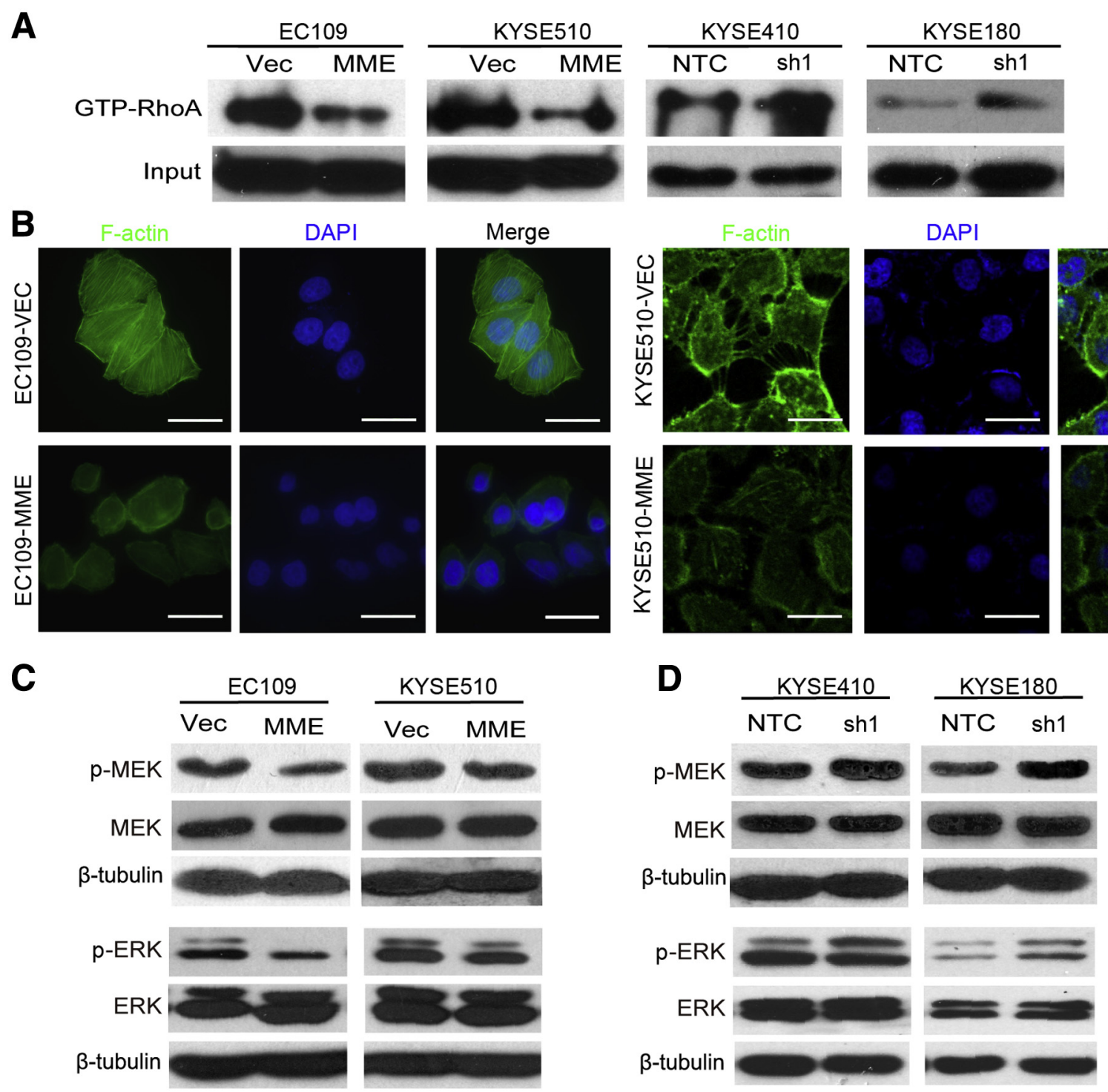

Figure 5 Membrane metalloendopeptidase (MME) suppresses FAK-RhoA axis. A: GST pulldown assay shows that the RhoA activation is inhibited in MMEtransfected cells (MME-EC109 and MME-KYSE510), whereas RhoA is activated in MME-silencing KYSE410 and KYSE180. GST-RhoA-RBD fusion protein is used to bind the activated form of GTP-bound RhoA. B: Alexa-488 phalloidin staining for F-actin (green) in MME-transfected cells and vector controls. Nuclei were counterstained with DAPI (blue). C and D: Western blotting shows the levels of phosphorylated MEK and ERK protein in MME overexpressed (C) or silenced (D) cells. $\beta$-Tubulin was used as a loading control. Scale bars $=20 \mu \mathrm{m}$. NTC, nontarget control.

reverse validation was also performed in MME silenced cells (Figure 5D).

\section{MME Inhibits ESCC Metastasis in Vivo}

Because in vitro assays showed that MME could suppress ESCC cell motility, animal pulmonary metastasis experiments were performed by injecting MME-transfected EC109 cells into Nu/Nu BALB/c mice via the tail vein. Tumor nodules that formed in the lungs were counted, and the number of nodules deceased significantly in the MME-EC109 group $(P<0.05)$ (Figure $6, \mathrm{~A}$ and B). Similar results were observed in the KYSE510-MME cell group (Figure 6C). EC109 derivative cells (MME and vector control) were inoculated via footpads of the mice to establish the lymph node metastasis animal models. Two months later, the popliteal lymph nodes were isolated and fixed for further examination (Figure 6D). Hematoxylin and eosin, and human CK staining results demonstrated that tumor cells were observed in 5 of 5 in the vector group versus only 2 of 5 in the MME group (Figure 6E).

\section{Discussion}

Tumor metastasis is an important cause of human cancerrelated deaths. ${ }^{21,22}$ The poor clinical outcome of ESCC is 

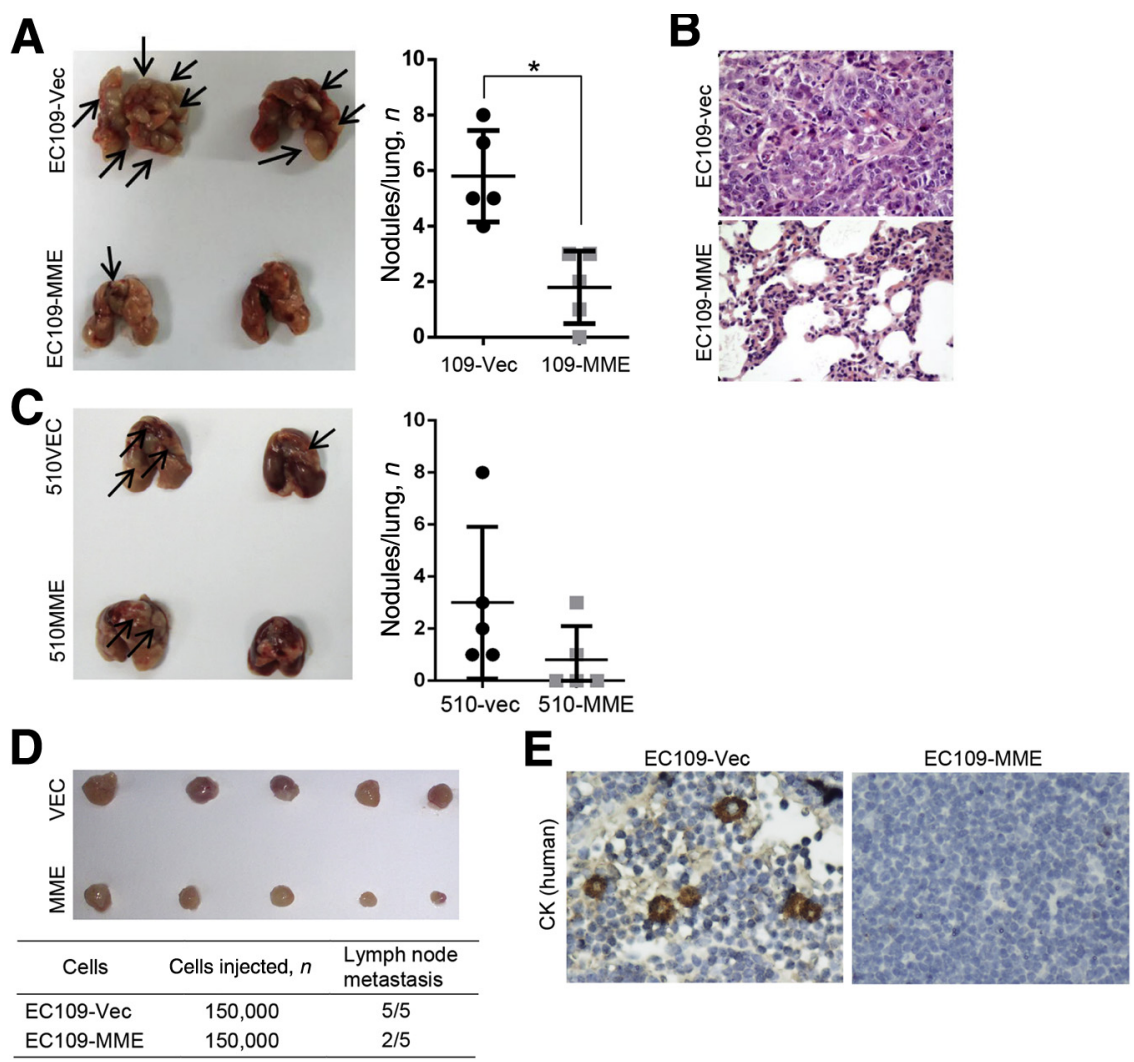

Figure 6 Membrane metalloendopeptidase (MME) inhibits tumor invasiveness in vivo. A: Representative pictures of lungs isolated from the mice inoculated with MME-EC109 and vector control cells via tail vein (arrows indicate nodules). Summary of nodules formed on the lungs is shown. B: Hematoxylin and eosin staining of mice lungs of the MME-EC109 and vector control groups. C: Representative pictures of lungs isolated from the mice inoculated with MME-KYSE510 and vector control cells via tail vein (arrows indicate nodules). Summary of nodules formed on the lungs is shown. D: Representative images of lymph nodes of the mice injected with MME-EC109 or vector control cells via footpad. The lymph nodes were stained with hematoxylin and eosin, and positive lymph nodes were counted. E: Lymph nodes invaded by tumor cells were confirmed by human CK (Pan) staining. ${ }^{*} P<0.05$. Original magnification: $\times 200(B) ; \times 400(E)$. mainly attributed to its nature to metastasize and invade easily. It has been reported that skipped metastasis was observed in $60 \%$ of early esophageal cancer patients, ${ }^{23}$ making it urgent to uncover the molecular mechanisms of ESCC metastasis, ${ }^{24}$ and identify and characterize molecules that are responsible for ESCC metastasis.

MME is a cell surface zinc metalloprotease. It is widely expressed in a variety of normal cell types. First identified as an oncogene in leukemia, ${ }^{4}$ it has been studied in several different types of cancer. ${ }^{25}$ It seems that the function of MME in cancer is cell-type-dependent: In colorectal cancer patients, tumoral MME expression has been substantially associated with liver metastasis. ${ }^{26}$ MME-positive expression correlates with tumor progression in pancreatic endocrine tumor $^{27}$ and malignant melanoma. ${ }^{28}$ In ovarian cancer, however, low MME expression may indicate a poor outcome (response to platinum) for patients. ${ }^{29}$ In prostate cancer, a better prognosis was predicted in the MMEpositive group. ${ }^{10,30}$ Conflicting results have been reported in bladder carcinoma: MME higher expression was reported in noninvasive carcinomas by one study, ${ }^{31}$ however, its upregulation facilitated tumor invasion and dissemination in another study. ${ }^{32}$

In this study, down-regulation of MME was markedly associated with advanced clinical stage $(P=0.04)$, lymph node metastasis $(P=0.024)$, and poorer prognosis $(P<0.001)$. Multivariate analyses demonstrated that MME down-regulation was an independent prognostic factor for overall survival. In vitro and in vivo assays proved that MME could suppress ESCC cell motility, but had no influence on ESCC cell proliferation.

To find the mechanism responsible for metastasis inhibition, a cell adhesion assay was performed in which MME overexpression was found to weaken cell adhesion ability. In most epithelia, FAK is a mediator for transducing signals at the focal adhesion complex facilitating cell migration. ${ }^{33,34}$ Consistent with a previous report, ${ }^{18}$ MME overexpression led to a decreased phosphorylation of FAK (Tyr397); however, the total FAK protein level remained unchanged, suggesting that MME overexpression inhibited FAK activation.

The Rho family of GTPases is composed of many small ubiquitous signaling $G$ proteins that bind to GTP and hydrolyze it to GDP. ${ }^{35}$ They act as switches: when binding to GTP, they are active and associate with a variety of target proteins that regulate intracellular actin dynamics needed for cell movement. Canonical members of the Rho family include RhoA, Rac1, and Cdc42. ${ }^{16,36}$ As adherence junction processes including formation, stability, and dissolution are regulated by Rho family GTPases ${ }^{37}$ and deletion of FAK in mice destabilizes RhoA and Rac1 activities, ${ }^{20}$ activated RhoA was tested in ESCC and RhoA activation was found to change accordingly with MME overexpression or knockdown in ESCC tumor cells. F-actin was also influenced as anticipated because actin cytoskeleton is a major target of Rho GTPases, which serve as a molecular switch on a variety of signaling pathways. ${ }^{38}$ Downstream 
MEK/ERK activation that is involved in tumor metastasis ${ }^{39}$ was also inhibited by MME overexpression.

In summary, our studies demonstrate the metastasissuppressor role of MME in ESCC and underscore the importance of FAK-RhoA axis regulated by MME to inhibit the tumor cell dissemination. Although MME plays multifaceted roles in various tumors, it suppresses ESCC tumor cell motility as shown in this study. Taken together, these findings shed light on complicated mechanisms of cancer dissemination in ESCC.

\section{Acknowledgments}

We thank Prof. G. Srivastava (Department of Pathology, The University of Hong Kong, Hong Kong, China) for the HKESC1, EC18, and EC109 cell lines; and Prof. G.S. Tsao (Department of Anatomy, The University of Hong Kong) for the immortalized nonmalignant esophageal cell line.

\section{Supplemental Data}

Supplemental material for this article can be found at http://doi.org/10.1016/j.ajpath.2019.04.007.

\section{References}

1. Noone AM, Cronin KA, Altekruse SF, Howlader N, Lewis DR, Petkov VI, Penberthy L: Cancer incidence and survival trends by subtype using data from the Surveillance Epidemiology and End Results Program, 1992-2013. Cancer Epidemiol Biomarkers Prev 2017, 26:632-641

2. Chen W, Zheng R, Baade PD, Zhang S, Zeng H, Bray F, Jemal A, Yu XQ, He J: Cancer statistics in China, 2015. CA Cancer J Clin 2016, 66:115-132

3. McCormack VA, Menya D, Munishi MO, Dzamalala C, Gasmelseed N, Leon Roux M, Assefa M, Osano O, Watts M, Mwasamwaja AO, Mmbaga BT, Murphy G, Abnet CC, Dawsey SM, Schuz J: Informing etiologic research priorities for squamous cell esophageal cancer in Africa: a review of setting-specific exposures to known and putative risk factors. Int J Cancer 2017, 140:259-271

4. Barker PE, Shipp MA, D'Adamio L, Masteller EL, Reinherz EL: The common acute lymphoblastic leukemia antigen gene maps to chromosomal region 3 (q21-q27). J Immunol 1989, 142:283-287

5. Depondt C, Donatello S, Rai M, Wang FC, Manto M, Simonis N, Pandolfo M: MME mutation in dominant spinocerebellar ataxia with neuropathy (SCA43). Neurol Genet 2016, 2:e94

6. Sahli S, Stump B, Welti T, Schweizer WB, Diederich F, BlumKaelin D, Aebi JD, Böhm H-J: A new class of inhibitors for the metalloprotease neprilysin based on a central imidazole scaffold. Helv Chim Acta 2005, 88:707-730

7. Wood BL, Arroz M, Barnett D, DiGiuseppe J, Greig B, Kussick SJ, Oldaker T, Shenkin M, Stone E, Wallace P: 2006 Bethesda International Consensus recommendations on the immunophenotypic analysis of hematolymphoid neoplasia by flow cytometry: optimal reagents and reporting for the flow cytometric diagnosis of hematopoietic neoplasia. Cytometry B Clin Cytom 2007, 72 Suppl 1:S14-S22

8. Choi WW, Weisenburger DD, Greiner TC, Piris MA, Banham AH, Delabie J, Braziel RM, Geng H, Iqbal J, Lenz G, Vose JM, Hans CP, Fu K, Smith LM, Li M, Liu Z, Gascoyne RD, Rosenwald A, Ott G, Rimsza LM, Campo E, Jaffe ES, Jaye DL, Staudt LM, Chan WC: A new immunostain algorithm classifies diffuse large B-cell lymphoma into molecular subtypes with high accuracy. Clin Cancer Res 2009, 15:5494-5502

9. Mizerska-Kowalska M, Bojarska-Junak A, Jakubowicz-Gil J, Kandefer-Szerszen M: Neutral endopeptidase (NEP) is differentially involved in biological activities and cell signaling of colon cancer cell lines derived from various stages of tumor development. Tumour Biol 2016, 37:13355-13368

10. Dai J, Shen R, Sumitomo M, Goldberg JS, Geng Y, Navarro D, Xu S, Koutcher JA, Garzotto M, Powell CT, Nanus DM: Tumor-suppressive effects of neutral endopeptidase in androgen-independent prostate cancer cells. Clin Cancer Res 2001, 7:1370-1377

11. Makretsov NA, Hayes M, Carter BA, Dabiri S, Gilks CB, Huntsman DG: Stromal CD10 expression in invasive breast carcinoma correlates with poor prognosis, estrogen receptor negativity, and high grade. Mod Pathol 2007, 20:84-89

12. Li Y, Chen L, Nie CJ, Zeng TT, Liu H, Mao X, Qin Y, Zhu YH, Fu L, Guan XY: Downregulation of RBMS3 is associated with poor prognosis in esophageal squamous cell carcinoma. Cancer Res 2011, 71:6106-6115

13. Watkins SJ, Borthwick GM, Arthur HM: The H9C2 cell line and primary neonatal cardiomyocyte cells show similar hypertrophic responses in vitro. In Vitro Cell Dev Biol Anim 2010, 47:125-131

14. Ponda MP, Breslow JL: Serum stimulation of CCR7 chemotaxis due to coagulation factor XIIa-dependent production of high-molecularweight kininogen domain 5. Proc Natl Acad Sci U S A 2016, 113: E7059-E7068

15. Liebl J, Weitensteiner SB, Vereb G, Takács L, Fürst R, Vollmar AM, Zahler S: Cyclin-dependent kinase 5 regulates endothelial cell migration and angiogenesis. J Biol Chem 2010, 285:35932-35943

16. Parsons JT, Horwitz AR, Schwartz MA: Cell adhesion: integrating cytoskeletal dynamics and cellular tension. Nat Rev Mol Cell Biol 2010, 11:633

17. Corey SJ, Yu J: Cancer cell migration: when red light switched to green. Asian J Androl 2011, 13:177-178

18. Sumitomo M, Shen R, Walburg M, Dai J, Geng Y, Navarro D, Boileau G, Papandreou CN, Giancotti FG, Knudsen B, Nanus DM: Neutral endopeptidase inhibits prostate cancer cell migration by blocking focal adhesion kinase signaling. J Clin Invest 2000, 106: $1399-1407$

19. Sulzmaier FJ, Jean C, Schlaepfer DD: FAK in cancer: mechanistic findings and clinical applications. Nat Rev Cancer 2014, 14:598-610

20. Schmidt TT, Tauseef M, Yue L, Bonini MG, Gothert J, Shen T-L, Guan J-L, Predescu S, Sadikot R, Mehta D: Conditional deletion of FAK in mice endothelium disrupts lung vascular barrier function due to destabilization of RhoA and Rac1 activities. Am J Physiol Lung Cell Mol Physiol 2013, 305:L291-L300

21. Rankin EB, Giaccia AJ: Hypoxic control of metastasis. Science 2016, $352: 175-180$

22. Massague J, Obenauf AC: Metastatic colonization by circulating tumour cells. Nature 2016, 529:298-306

23. Cho JW, Choi SC, Jang JY, Shin SK, Choi KD, Lee JH, Kim SG, Sung JK, Jeon SW, Choi IJ, Kim GH, Jee SR, Lee WS, Jung H-Y, Korean ESDSG: Lymph node metastases in esophageal carcinoma: an endoscopist's view. Clin Endosc 2014, 47:523-529

24. Chiang AC, Massagué J: Molecular basis of metastasis. N Engl J Med 2008, 359:2814-2823

25. Maguer-Satta V, Besancon R, Bachelard-Cascales E: Concise review: neutral endopeptidase (CD10): a multifaceted environment actor in stem cells, physiological mechanisms, and cancer. Stem Cells 2011, 29:389-396

26. Fujimoto Y, Nakanishi Y, Sekine S, Yoshimura K, Akasu T, Moriya Y, Shimoda T: CD10 expression in colorectal carcinoma correlates with liver metastasis. Dis Colon Rectum 2005, 48:1883-1889

27. Deschamps L, Handra-Luca A, O'Toole D, Sauvanet A, Ruszniewski P, Belghiti J, Bedossa P, Couvelard A: CD10 expression 
in pancreatic endocrine tumors: correlation with prognostic factors and survival. Hum Pathol 2006, 37:802-808

28. Oba J, Nakahara T, Hayashida S, Kido M, Xie L, Takahara M, Uchi H, Miyazaki S, Abe T, Hagihara A, Moroi Y, Furue M: Expression of CD10 predicts tumor progression and unfavorable prognosis in malignant melanoma. J Am Acad Dermatol 2011, 65:1152-1160

29. Ffrench B, Gasch C, Hokamp K, Spillane C, Blackshields G, Mahgoub TM, Bates M, Kehoe L, Mooney A, Doyle R, Doyle B, O'Donnell D, Gleeson N, Hennessy BT, Stordal B, O'Riain C, Lambkin H, O’Toole S, O'Leary JJ, Gallagher MF: CD10(-)/ALDH(-) cells are the sole cisplatin-resistant component of a novel ovarian cancer stem cell hierarchy. Cell Death Dis 2017, 8:e3128

30. Osman I, Dai J, Mikhail M, Navarro D, Taneja SS, Lee P, Christos P, Shen R, Nanus DM: Loss of neutral endopeptidase and activation of protein kinase B (Akt) is associated with prostate cancer progression. Cancer 2006, 107:2628-2636

31. Bircan S, Candir O, Kapucuoglu N, Serel TA, Ciris M, Karahan N: CD10 expression in urothelial bladder carcinomas: a pilot study. Urol Int 2006, 77:107-113

32. Abdou AG: CD10 expression in tumour and stromal cells of bladder carcinoma: an association with bilharziasis. APMIS 2007, 115: $1206-1218$
33. Schaller MD, Hildebrand JD, Shannon JD, Fox JW, Vines RR, Parsons JT: Autophosphorylation of the focal adhesion kinase, pp125FAK, directs SH2-dependent binding of pp60src. Mol Cell Biol 1994, 14:1680-1688

34. McLean GW, Carragher NO, Avizienyte E, Evans J, Brunton VG, Frame MC: The role of focal-adhesion kinase in cancer-a new therapeutic opportunity. Nat Rev Cancer 2005, 5:505-515

35. Chiba S, Enami T, Ogawa S, Sakata-Yanagimoto M: G17V RHOA: genetic evidence of GTP-unbound RHOA playing a role in tumorigenesis in T cells. Small GTPases 2015, 6:100-103

36. Jaffe AB, Hall A: Rho GTPases: biochemistry and biology. Annu Rev Cell Dev Biol 2005, 21:247-269

37. Thiery JP: Epithelial-mesenchymal transitions in tumour progression. Nat Rev Cancer 2002, 2:442-454

38. Rajakyla EK, Vartiainen MK: Rho, nuclear actin, and actin-binding proteins in the regulation of transcription and gene expression. Small GTPases 2014, 5:e27539

39. Tong M, Chan KW, Bao JY, Wong KY, Chen JN, Kwan PS, Tang KH, Fu L, Qin YR, Lok S, Guan XY, Ma S: Rab25 is a tumor suppressor gene with antiangiogenic and anti-invasive activities in esophageal squamous cell carcinoma. Cancer Res 2012, 72: 6024-6035 